03

\title{
Влияние наклонного электростатического поля на неустойчивость Кельвина-Гельмгольца при течении жидкого диэлектрика и газа
}

\author{
(C) В.М. Коровин \\ Научно-исследовательский институт механики Московского государственного университета им. М.В. Ломоносова, \\ 119192 Москва, Россия \\ e-mail: verazhan@yandex.ru
}

(Поступило в Редакцию 20 ноября 2015 г. В окончательной редакции 4 марта 2017 г.)

В линейной постановке на базе уравнений и граничных условий гидродинамики идеальной несжимаемой жидкости и электростатики изучен эффект, вызываемый действием поверхностных пондеромоторных сил на неустойчивость Кельвина-Гельмгольца. Выписаны в виде неравенств условия, которым должны удовлетворять величины определяющих параметров задачи, чтобы неустойчивое при отсутствии электрического поля течение переходило в устойчивый режим после наложения горизонтального электрического поля. Показано, что на границе устойчивости длина волны наиболее неустойчивой моды не зависит от пондеромоторных сил. Установлено, что в случае жидкости с большой диэлектрической проницаемостью имеется устойчивый режим течения, для которого условие устойчивости отличается лишь малыми безразмерными величинами от условия устойчивости заряженной поверхности покоящегося жидкого проводника, граничащего с неподвижным газом.

DOI: 10.21883/JTF.2017.09.44902.1674

\section{Введение}

Система уравнений и граничных условий электрогидродинамики, описываемая моделью Тейлора-Мелчера $[1,2]$, представляет результаты экспериментальных и теоретических исследований, проведенных во второй половине XX в. Эта модель учитывает как уход из рассматриваемого объема жидкого диэлектрика свободных электрических зарядов, образующихся в результате различных физико-химических процессов в объеме и на его границе, так и поступление в этот объем свободных зарядов извне.

Наряду с моделью Тейлора-Мелчера в электрогидродинамике используется более простая модель однородного по температуре (и другим физическим характеристикам) несжимаемого жидкого диэлектрика, не содержащего свободных электрических зарядов (см., например, работы [3-7]). Теоретические основы такого подхода были заложены в конце XIX в. [8]. В этой модели запись пондеромоторных сил является частным случаем общих формул электродинамики сплошных сред [9].

В однородном несжимаемом жидком диэлектрике при отсутствии свободных электрических зарядов объемные пондеромоторные силы отсутствуют. Ввиду этого в рамках такой модели электрическое поле оказывает силовое воздействие на жидкость лишь за счет пондеромоторных сил, локализованных на поверхности раздела с газом или с жидкостью, имеющей другую диэлектрическую проницаемость.

Известно, что при включении и последующем квазистатическом увеличении напряженности однородного электрического поля, перпендикулярного горизонтальной свободной поверхности покоящегося жидкого диэлектрика, при превышении критической величины поля реализуется гидростатическое состояние жидкости с неплоской свободной поверхностью. В отличие от этого поверхностные пондеромоторные силы, порождаемые статическим параллельным полем, оказывают стабилизирующее действие на вызываемые различными факторами неустойчивости поверхностей раздела диэлектрическая жидкость-газ.

При движении тонкой плоской струи жидкости в потоке воздуха, имеющем другую по величине, но одинаково направленную скорость, при наличии продольного электрического поля неустойчивость наступает при более высокой скорости струи [3]. При капиллярном распаде цилиндрической струи, находящейся в продольном электрическом поле и окруженной газом с постоянным давлением, область неустойчивости (в плоскости параметров „волновое число - квадрат частоты“) уменьшается за счет смещения ее правой границы в сторону более длинных волн [4]. В рамках нелинейной теории показано, что продольное поле препятствует вызываемому капиллярными силами разрыву тонкой пленки невязкой диэлектрической жидкости [5]. Достаточно сильное продольное поле гасит растущие возмущения в сверхтонком (толщиной 100-1000 А) плоском слое диэлектрической жидкости, динамика которой наряду с капиллярными, вязкими и пондеромоторными силами определяется также расклинивающим давлением, создаваемым силами Ван-дер-Ваальса [6].

В отличие от имеющихся публикаций в настоящей работе исследовано влияние наклонного электрического поля на неустойчивость стационарного течения с одинаково направленными скоростями расположенных друг над другом газа и диэлектрической жидкости, имеющих разрыв скорости на горизонтальной поверхности раздела. 


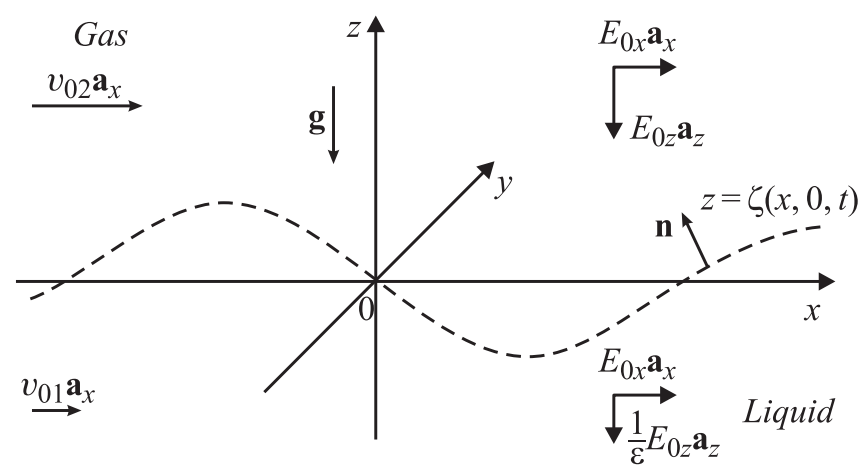

Рис. 1. Геометрия задачи и обозначения.

\section{Определяющие уравнения и граничные условия}

В достаточно большой области трехмерного пространства рассматриваются горизонтальные потоки невязкой диэлектрической жидкости и расположенного над ней газа. Предполагается, что вдали от поверхности раздела сред в газе с помощью внешних устройств создано наклонное электростатическое поле $\mathbf{E}_{0}-$ рис. 1 . В жидкости и в газе свободные электрические заряды отсутствуют. Поле $E_{0}$, естественно, не превышает пробивного значения $E_{b}$. Для сухого воздуха $E_{b}=30 \mathrm{kV} / \mathrm{cm}$ [8]. Далее в качестве основной системы единиц используется система CGS.

Введем декартову систему координат $x, y, z$, у которой ось $x$ параллельна горизонтальной составляющей электрического поля $\mathbf{E}_{0}$, а ось $z$ направлена вертикально. Обозначим через $\mathbf{a}_{x}, \mathbf{a}_{y}, \mathbf{a}_{z}$ соответствующие орты. Далее индексами $j=1,2$ отмечаются физические величины, относящиеся к жидкости $(j=$ $=1)$ и к газу $(j=2)$. Пусть уравнение $z=\xi$ представляет форму поверхности раздела жидкости и газа. Ввиду наличия случайных возмущений, неизбежно возникающих в экспериментах, $\xi=\zeta(x, y, t)$, где $t-$ время.

Распределения электрических полей $\mathbf{E}_{j}=-\nabla u_{j}$ $\times(x, y, z, t)$ описываются уравнениями и граничными условиями электростатики диэлектриков

$$
\begin{aligned}
& \nabla^{2} u_{j}=0, \nabla=\mathbf{a}_{x} \frac{\partial}{\partial x}+\mathbf{a}_{y} \frac{\partial}{\partial y}+\mathbf{a}_{z} \frac{\partial}{\partial z}, j=1,2, \\
& \text { при } z=\xi(z, y, t): \quad u_{1}=u_{2}, \quad \varepsilon \frac{\partial u_{1}}{\partial n}=\frac{\partial u_{2}}{\partial n}, \\
& \text { при } z \rightarrow+\infty: \quad \nabla u_{2} \rightarrow-\mathbf{E}_{0}=-E_{0 x} \mathbf{a}_{x}-E_{0 z} \mathbf{a}_{z},
\end{aligned}
$$

где $\varepsilon-$ диэлектрическая проницаемость жидкости, а $\mathbf{n}(x, y, t)$ - единичный вектор нормали к поверхности раздела, направленный в сторону газа:

$\mathbf{n}(x, y, t)=\frac{1}{\sqrt{1+\left(\frac{\partial \xi}{\partial x}\right)^{2}+\left(\frac{\partial \xi}{\partial y}\right)^{2}}}\left(-\frac{\partial \xi}{\partial x} \mathbf{a}_{x}-\frac{\partial \xi}{\partial y} \mathbf{a}_{y}+\mathbf{a}_{z}\right)$.
Течения сред описываются уравнениями неразрывности и уравнениями Эйлера

$$
\begin{aligned}
\operatorname{div} \mathbf{v}_{j} & =0, \quad j=1,2, \\
\rho_{j}\left[\frac{\partial \mathbf{v}_{j}}{\partial t}+\left(\mathbf{v}_{j}, \nabla\right) \mathbf{v}_{j}\right] & =-\nabla p_{j}+\rho_{j} \mathbf{g}, \quad j=1,2 .
\end{aligned}
$$

Здесь $\mathbf{v}_{j}(x, y, z, t)=\left(v_{j x}, v_{j y}, v_{j z}\right)-$ скорость, $\rho_{j}-$ плотность, $p_{j}(x, y, z, t)$ - давление, $\mathbf{g}$ - ускорение силы тяжести. Предполагается, что течения потенциальны.

Два кинематических условия и динамическое условие на поверхности раздела газ-жидкость имеют вид

$$
\begin{gathered}
\text { при } z=\xi(x, y, t): \frac{\partial \xi}{\partial t}+\mathbf{v}_{j} \nabla \xi=v_{j z}, j=1,2, \\
\text { при } z=\xi(x, y, t): p_{1}-p_{2}=\alpha \operatorname{div} \mathbf{n} \\
-\frac{\varepsilon-1}{8 \pi}\left[\left(\nabla u_{1}\right)^{2}+(\varepsilon-1)\left(\frac{\partial u_{1}}{\partial n}\right)^{2}\right],
\end{gathered}
$$

где $\alpha-$ коэффициент поверхностного натяжения.

Пропорциональное $\varepsilon-1$ слагаемое в правой части динамического условия (7) представляет вклад поверхностных пондеромоторных сил [9].

Предполагается, что вдали от поверхности раздела сред жидкость и газ движутся горизонтально с постоянными скоростями $v_{0 j} \mathbf{a}_{x} t$,

$$
\begin{array}{ll}
\text { при } z \rightarrow+\infty: & \mathbf{v}_{1} \rightarrow v_{01} \mathbf{a}_{x}, \\
\text { при } z \rightarrow+\infty: & \mathbf{v}_{2} \rightarrow v_{02} \mathbf{a}_{x},
\end{array}
$$

причем $0<v_{01}<v_{0.2}$.

Задача (1)-(8) имеет точное решение

$$
\begin{gathered}
\xi_{0}=0, \quad u_{01}(x, z)=-E_{0 x} x-\frac{1}{\varepsilon} E_{0 z} z, \\
u_{02}(x, z)=-E_{0 x} x-E_{0 z} z, \\
\mathbf{v}_{01}=v_{01} \mathbf{a}_{x}, \quad \mathbf{v}_{02}=v_{02} \mathbf{a}_{x}, \\
p_{01}(z)=p_{0}-\rho_{1} g z-\frac{\varepsilon-1}{8 \pi}\left[E_{0 x}^{2}+\frac{1}{\varepsilon} E_{0 z}^{2}\right], \\
p_{02}(z)=p_{0}-\rho_{2} g z,
\end{gathered}
$$

где $p_{0}$ - постоянное давление.

При исследовании неустойчивости Кельвина-Гельмгольца в отсутствие электрического поля [10] выражения (9) с $\mathbf{E}_{0}=0$ представляют основное течение.

\section{Постановка задачи}

Для исследования устойчивости точного стационарного решения (9) по отношению к бесконечно малым возмущениям применим стандартный подход [11]. Предварительно с целью упрощения дальнейших выкладок перейдем к системе координат, движущейся со скоростью $\mathbf{v}_{01}$, сохраняя при этом обозначения $x, y, z$ для координат и полагая $\mathbf{v}_{0}=\mathbf{v}_{02}-\mathbf{v}_{01}$. 
Наложим на решение (9) малые возмущения:

при $z<\zeta(x, y, t): u_{1}(x, y, z, t)=u_{01}(x, z)+\psi_{1}(x, y, z, t)$,

$$
\begin{gathered}
\mathbf{v}_{1}(x, y, z, t)=\nabla \varphi_{1}(x, y, z, t), \\
p_{1}(x, y, z, t)=p_{01}(z)+q_{1}(x, y, z, t),
\end{gathered}
$$

при $z>\zeta(x, y, t): u_{2}(x, y, z, t)=u_{02}(x, z)+\psi_{2}(x, y, z, t)$,

$$
\begin{aligned}
\mathbf{v}_{2}(x, y, z, t) & =\mathbf{v}_{0}+\nabla \varphi_{2}(x, y, z, t), \\
p_{2}(x, y, z, t) & =p_{02}(z)+q_{2}(x, y, z, t) .
\end{aligned}
$$

Считается, что возмущение $\xi(x, y, t)$ плоской поверхности раздела $z=0$ таково, что возмущенная поверхность раздела удовлетворяет требованию малости наклонов [12]. Физически данное требование означает, что локальное вертикальное отклонение $|\xi(x, y, t)|$ возмущенной поверхности от горизонтального положения $z=0$ много меньше характерной протяженности горба или впадины. В уравнении движения (5) в таком случае инерционный член $\left(\mathbf{v}_{j}, \nabla\right) \mathbf{v}_{j}$ мал по сравнению с линейным членом $\partial \mathbf{v}_{j} / \partial t$ и ввиду этого опускается.

Подстановка выражений (10), (11) в уравнения (1), (4), (5) приводит к результату

$$
\begin{gathered}
\nabla^{2} \psi_{j}=0, \quad \nabla^{2} \varphi_{j}=0, \quad j=1,2, \\
q_{1}=-\rho_{1} \frac{\partial \varphi_{1}}{\partial t}, \quad q_{2}=-\rho_{2}\left(\frac{\partial \varphi_{2}}{\partial t}+v_{0} \frac{\partial \varphi_{2}}{\partial x}\right) .
\end{gathered}
$$

После линеаризации граничных условий электростатики (2) и гидродинамики (6), (7), проведенной с учетом выражений (10), (11), (13), получаем

при

$$
\begin{gathered}
z=0: \quad \psi_{2}-\psi_{1}=\frac{\varepsilon-1}{\varepsilon} E_{0 z} \zeta \\
\frac{\partial \psi_{2}}{\partial z}-\varepsilon \frac{\partial \psi_{1}}{\partial z}=(\varepsilon-1) E_{0 x} \frac{\partial \xi}{\partial x}, \\
\rho_{1} \frac{\partial \varphi_{1}}{\partial t}-\rho_{2}\left(\frac{\partial \varphi_{2}}{\partial t}+v_{0} \frac{\partial \varphi_{2}}{\partial x}\right)+g \xi\left(\rho_{1}-\rho_{2}\right) \\
-\alpha\left(\frac{\partial^{2} \zeta}{\partial x^{2}}+\frac{\partial^{2} \zeta}{\partial y^{2}}\right)+\frac{\varepsilon-1}{4 \pi}\left(E_{0 x} \frac{\partial \psi_{1}}{\partial x}-E_{0 z} \frac{\partial \psi_{1}}{\partial z}\right. \\
\left.+\frac{\varepsilon-1}{\varepsilon} E_{0 x} E_{0 z} \frac{\partial \xi}{\partial x}\right)=0 \\
\frac{\partial \xi}{\partial t}=\frac{\partial \varphi_{1}}{\partial z}, \quad \frac{\partial \xi}{\partial t}+v_{0} \frac{\partial \xi}{\partial x}=\frac{\partial \varphi_{2}}{\partial z}
\end{gathered}
$$

В силу (3), (8), (10), (11) имеем при

$$
z \rightarrow+\infty: \quad \nabla \psi_{2} \rightarrow 0, \quad \nabla \varphi_{2} \rightarrow 0
$$

при

$$
z \rightarrow-\infty: \quad \nabla \varphi_{1} \rightarrow 0 .
$$

Далее рассматривается задача (12), (14), (15).

\section{Дисперсионное уравнение}

Исследование поведения с ростом времени решения сформулированной линейной задачи, зависящего от $x, y, z, t$, проведем с использованием метода нормальных мод [10]. Положим

$$
\begin{aligned}
& {\left[\xi(x, y, t), \psi_{j}(x, y, z, t), \varphi_{j}(x, y, z, t)\right]=\left[Z, \Psi_{j}(z), \Phi_{j}(z)\right]} \\
& \quad \times \exp \left\{\left[i\left(k_{x} x+k_{y} y-\omega\left(k_{x}, k_{y}\right) t\right)\right]\right\}, \quad j=1,2, \quad(16)
\end{aligned}
$$

где $Z-$ константа, $i-$ мнимая единица, $\mathbf{k}=$ $=k_{x} \mathbf{a}_{x}+k_{y} \mathbf{a}_{y},-\infty<k_{x}<+\infty,-\infty<k_{y}<+\infty, k_{x}^{2}+$ $+k_{y}^{2} \neq 0$ - волновой вектор (действительный параметр), а функция $\omega=\omega\left(k_{x}, k_{y}\right)$ подлежит нахождению из уравнений и граничных условий.

После подстановки выражений (16) в уравнения Лапласа (12) получаем

$$
\begin{gathered}
\frac{d^{2} \Psi_{j}}{d z^{2}}-k^{2} \Psi_{j}=0, \quad \frac{d^{2} \Phi_{j}}{d z^{2}}-k^{2} \Phi_{j}=0, \\
j=1,2, \quad k=\sqrt{k_{x}^{2}+k_{y}^{2}} .
\end{gathered}
$$

Граничные условия (14) принимают вид

$$
\begin{gathered}
\text { при } z=0: \quad \Psi_{2}-\Psi_{1}=\frac{\varepsilon-1}{\varepsilon} E_{0 z} Z \\
\frac{d \Psi_{2}}{d z}-\varepsilon \frac{d \Psi_{1}}{d z}=i(\varepsilon-1) Z E_{0 x} k_{x}, \\
i \omega\left(\rho_{2} \Phi_{2}-\rho_{1} \Phi_{1}\right)-i k_{x} \rho_{2} v_{0} \Phi_{2}+\left[g\left(\rho_{1}-\rho_{2}\right)+\alpha k^{2}\right] Z \\
+\frac{\varepsilon-1}{4 \pi}\left[i k_{x} E_{0 x}\left(\Psi_{1}+\frac{\varepsilon-1}{\varepsilon} E_{0 z} Z\right)-E_{0 z} \frac{d \Psi_{1}}{d z}\right] \\
i \omega Z+\frac{d \Phi_{1}}{d z}=0, \\
i\left(k_{x} v_{0}-\omega\right) Z-\frac{d \Phi_{2}}{d z}=0 .
\end{gathered}
$$

Исчезающие при $z \rightarrow \pm \infty$ решения уравнений (17) имеют вид

$$
\begin{array}{ll}
\Psi_{1}=A_{1} \exp (k z), & \Psi_{2}=A_{2} \exp (-k z), \\
\Phi_{1}=B_{1} \exp (k z), & \Phi_{2}=B_{2} \exp (-k z),
\end{array}
$$

где $A_{1}, A_{2}, B_{1}, B_{2}$ - произвольные константы.

После подстановки выписанных решений в граничные условия (18) приходим к следующей системе уравнений относительно $A_{1}, A_{2}, B_{1}, B_{2}, Z$ :

$$
A_{1}-A_{2}+\frac{\varepsilon-1}{\varepsilon} E_{0 z} Z=0,
$$

$$
k\left(\varepsilon A_{1}+A_{2}\right)+i(\varepsilon-1) E_{0 x} k_{x} Z=0,
$$

$$
\begin{gathered}
\frac{\varepsilon-1}{4 \pi}\left(i k_{x} E_{0 x}-k E_{0 z}\right) A_{1}-i \rho_{1} \omega B_{1}-i \rho_{2}\left(k_{x} v_{0}-\omega\right) B_{2} \\
+\left[\alpha k^{2}+g\left(\rho_{1}-\rho_{2}\right)\right] Z=0,
\end{gathered}
$$




$$
\begin{gathered}
k B_{1}+i \omega Z=0, \\
k B_{2}-i\left(k_{x} v_{0}-\omega\right) Z=0 .
\end{gathered}
$$

Условие существования нетривиального решения системы однородных уравнений (19) - равенство нулю ее определителя

$$
\begin{gathered}
\left(\rho_{1}+\rho_{2}\right) \omega^{2}-2 k_{x} \rho_{2} v_{0} \omega+k_{x}^{2} \rho_{2} v_{0}^{2}-g k\left(\rho_{1}-\rho_{2}\right)-\alpha k^{3} \\
-m\left[\varepsilon\left(E_{0 x} k_{x}\right)^{2}-\left(E_{0 z} k\right)^{2}\right]=0, \quad m=\frac{(\varepsilon-1)^{2}}{4 \pi \varepsilon(\varepsilon+1)}
\end{gathered}
$$

представляет дисперсионное уравнение.

\section{Влияние пондеромоторных сил на течение}

Обращаясь к (20), легко найти корни дисперсионного уравнения

$$
\omega_{1,2}\left(k_{x}, k_{y}\right)=\frac{\rho_{2} v_{0}}{\rho_{1}+\rho_{2}} k_{x} \pm \sqrt{\frac{f\left(k_{x}, k_{y}\right)}{\rho_{1}+\rho_{2}}},
$$

где

$$
\begin{aligned}
f\left(k_{x}, k_{y}\right)= & \alpha\left(k_{x}^{2}+k_{y}^{2}\right)^{3 / 2}-m E_{0 z}^{2}\left(k_{x}^{2}+k_{y}^{2}\right) \\
& +g\left(\rho_{1}-\rho_{2}\right)\left(k_{x}^{2}+k_{y}^{2}\right)^{1 / 2}+s k_{x}^{2}, \\
s & =m \varepsilon E_{0 x}^{2}-\frac{\rho_{1} \rho_{2} v_{0}^{2}}{\rho_{1}+\rho_{2}} .
\end{aligned}
$$

Из (16), (21) следует, что при заданных значениях определяющих параметров задачи моды с волновыми векторами $\mathbf{k}=\left(k_{x}, k_{y}\right)$, для которых $g\left(k_{x}, k_{y}\right)>0$, устойчивы. Если $f\left(k_{x}, k_{y}\right)<0$, то двузначная комплексная функция $\omega\left(k_{x}, k_{y}\right)$ имеет ветвь, на которой $\operatorname{Im} \omega\left(k_{x}, k_{y}\right)>0$. Ввиду этого моды, соответствующие таким $k_{x}, k_{y}$, экспоненциально растут по времени. В случае $f\left(k_{x}, k_{y}\right)=0$ мода нейтрально устойчива.

Таким образом, при заданных $v_{0}, E_{0 x}, E_{0 z}$ течение сред с конкретными физическими свойствами устойчиво, если $f\left(k_{x}, k_{y}\right)>0$ при $-\infty<k_{x}<+\infty,-\infty<k_{y}<+\infty$, $k_{x}^{2}+k_{y}^{2} \neq 0$.

С целью упрощения исследования функции $f\left(k_{x}, k_{y}\right)$ перейдем в плоскости переменных $k_{x}, k_{y}$ к полярной системе координат $k, \vartheta$ :

$$
k_{x}=k \cos \vartheta, \quad k_{y}=k \sin \vartheta, \quad 0 \leq \vartheta \leq 2 \pi,
$$

В результате получаем

$$
\begin{gathered}
f\left(k_{x}, k_{y}\right)=k q(k, \vartheta), \\
q(k, \vartheta)=\alpha k^{2}+\left(s \cos ^{2} \vartheta-m E_{0 z}^{2}\right) k+g\left(\rho_{1}-\rho_{2}\right) .
\end{gathered}
$$

Поскольку функция $q(k, \vartheta)$ является периодической по $\vartheta$ с периодом $\pi$, для устойчивости течения требуется $q(k, \vartheta)>0$ при $0<k<\infty, 0 \leq \vartheta \leq \pi$.

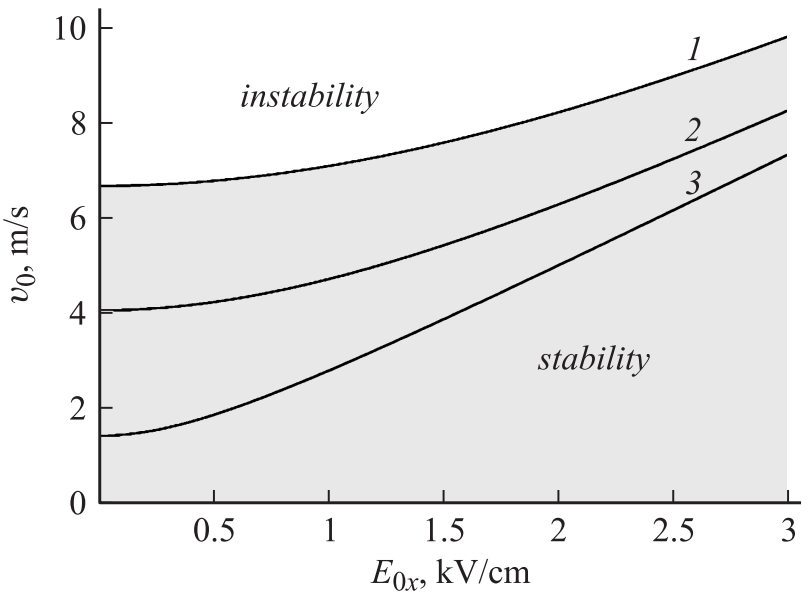

Рис. 2. Кривые нейтральной устойчивости. Линии 1-3 соответствуют значениям $E_{0 z}=0,19.5,24 \mathrm{kV} / \mathrm{cm}$.

При особом режиме течения, когда $s=0$, поверхность, представляемая уравнением $f=f\left(k_{x}, k_{y}\right)$, является поверхностью вращения. В этом случае предыдущее неравенство принимает вид

$$
\left.q\right|_{s=0}=\alpha k^{2}-m E_{0 z}^{2} k+g\left(\rho_{1}-\rho_{2}\right)>0 .
$$

Для выполнения этого условия требуется, чтобы дискриминант квадратного трехчлена $\left.q\right|_{s=0}$ был отрицателен.

Таким образом, течение устойчиво, если

$$
m^{2} E_{0 z}^{4}<4 \alpha g\left(\rho_{1}-\rho_{2}\right) .
$$

В предельном случае, при $\varepsilon \rightarrow \infty, \rho_{2} / \rho_{1} \rightarrow 0$ следует $E_{0 z}^{4}<64 \pi^{2} \alpha \rho_{1} g$. Это выражение совпадает с условием устойчивости граничащей с покоящимся газом горизонтальной поверхности неподвижного жидкого проводника, на которой находятся электрические заряды, создающие вертикальное электрическое поле $E_{0 z}=4 \pi \sigma_{0}$, где $\sigma_{0}-$ поверхностная плотность зарядов. Впервые явление неустойчивости для жидких проводников было исследовано Тонксом и Френкелем [9], [13], [14].

Таким образом, при особом режиме течения газа и жидкого диэлектрика, имеющего бо́льшую $(\varepsilon \gg 1)$ диэлектрическую проницаемость, условие устойчивости (22) отличается лишь безразмерными величинами порядков $\varepsilon^{-1}, \rho_{2} / \rho_{1} \ll 1$ от условия устойчивости заряженной плоской поверхности покоящегося жидкого проводника в поле тяжести.

Рассмотрим случай $s<0$. Обозначим $\tau=\cos ^{2} \vartheta$, тогда

$$
\begin{gathered}
q(k, \vartheta)=r(k, \tau)=\alpha k^{2}+\left(s \tau-m E_{0 z}^{2}\right) k+g\left(\rho_{1}-\rho_{2}\right), \\
0 \leq \tau \leq 1 .
\end{gathered}
$$

Выпишем дискриминант $D(k, \tau)$ квадратного трехчлена $r(k, \tau)$, зависящего от параметра $\tau$ :

$$
D(k, \tau)=\left(s \tau-m E_{0 z}^{2}\right)^{2}-4 \alpha g\left(\rho_{1}-\rho_{2}\right) .
$$


При $s<0$ имеем $d D / d \tau>0$, т. е. на отрезке $0 \leq \tau \leq 1$ при любом $k>0$ функция $D(k, \tau)$ возрастает с ростом $\tau$. Ввиду этого условие устойчивости течения записывается следующим образом:

$$
\left[\frac{\rho_{1} \rho_{2} v_{0}^{2}}{\rho_{1}+\rho_{2}}+m\left(E_{0 z}^{2}-\varepsilon E_{0 x}^{2}\right)\right]^{2}-4 \alpha g\left(\rho_{1}-\rho_{2}\right)<0 .
$$

В отсутствие электрического поля это выражение совпадает с условием устойчивости тангенциального разрыва в поле тяжести с учетом поверхностного натяжения [11].

Из (23) следует

$$
v_{0}<\left\{\frac{\rho_{1}+\rho_{2}}{\rho_{1} \rho_{2}}\left[\sqrt{4 \alpha g\left(\rho_{1}-\rho_{2}\right)}+m\left(\varepsilon E_{0 x}^{2}-E_{0 z}^{2}\right)\right]\right\}^{1 / 2} .
$$

В качестве примера рассмотрим относительное движение воздуха и воды. При температуре $20^{\circ} \mathrm{C}$ имеем $\rho_{1}=0.9982 \mathrm{~g} / \mathrm{cm}^{3}, \rho_{2}=1.205 \cdot 10^{-3} \mathrm{~g} / \mathrm{cm}^{3}, \alpha=$ $=72.8 \mathrm{dym} / \mathrm{cm}[15]$, а для перегнанной в вакууме воды $\varepsilon=78.3[16]$.

На рис. 2 в плоскости переменных $E_{0 x}, v_{0}$ при различных значениях $E_{0 z}$ показаны кривые нейтральной устойчивости, вдоль которых левая часть неравенства (23) обращается в нуль. Из графиков видно, что неустойчивое при $\mathbf{E}_{0}=0$ течение можно перевести в устойчивый режим путем наложения горизонтального поля достаточно высокой напряженности. Наличие вертикальной компоненты поля сужает диапазон разрыва скорости, в котором капиллярные силы и горизонтальная компонента электрического поля подавляют неустойчивость Кельвина-Гельмгольца.

Применительно к случаю $s<0$ с использованием выписанных выражений легко находится длина волны $\lambda_{m}=2 \pi / k_{m}, k_{m}=\sqrt{\alpha^{-1} g\left(\rho_{1}-\rho_{2}\right)}$ наиболее неустойчивой моды на границе устойчивости, когда выполнены условия $r(k, 1)=0,\left(m E_{0 z}-s\right)^{2}=4 \alpha g\left(\rho_{1}-\rho_{2}\right)$.

В случае устойчивого течения именно мода с длиной волны $\lambda_{m}$ первой теряет устойчивость при фиксированной величине $E_{0 x}$ и бесконечно малом увеличении хотя бы одного из определяющих параметров $E_{0 z}, v_{0}$. Отметим, что пондеромоторные силы не влияют на величину $\lambda_{m}$.

\section{Заключение}

В рамках систем уравнений и граничных условий гидродинамики идеальной несжимаемой жидкости и электростатики исследовано влияние поверхностных пондеромоторных сил на устойчивость стационарного течения газа и диэлектрической жидкости. Рассматриваемые среды движутся горизонтально в одном направлении при наличии заданного на поверхности их раздела разрыва скорости. Использована модель однородного по своим физическим характеристикам жидкого диэлектрика, температура которого постоянна. Свободных электрических зарядов в рассматриваемой системе нет. Предполагается, что в газе вдали от поверхности раздела задано наклонное однородное электростатическое поле.

Выведено квадратное относительно частоты дисперсионное уравнение. Изучено влияние заданных вертикальной и горизонтальной компонент электрического поля и величины разрыва скорости на расположение корней дисперсионного уравнения в плоскости, представляющей в общем случае комплексную частоту. Использование полярной системы координат при записи двумерного волнового вектора существенно упрощает исследование.

В результате проведенного анализа найдены в виде неравенств условия, которым должны удовлетворять величины определяющих параметров задачи, для того чтобы неустойчивое при отсутствии электрического поля течение после наложения горизонтального электрического поля переходило в устойчивый режим. Вертикальная компонента электрического поля всегда оказывает дестабилизирующее действие.

Установлено, что на границе устойчивости длина волны наиболее неустойчивой моды определяется гравитационными и капиллярными силами и не зависит от пондеромоторных сил.

Показано, что при выполнении некоторого равенства, в котором фигурируют квадрат заданного разрыва скорости и квадрат заданной горизонтальной компоненты электрического поля, возможен особый режим течения газа и жидкости с любой диэлектрической проницаемостью $\varepsilon$. В этом случае при $\varepsilon \gg 1$ найденное условие устойчивости, определяющее верхнюю границу величины вертикальной компоненты электрического поля, отличается лишь малыми безразмерными величинами от найденного Тонксом и Френкелем условия устойчивости, граничащей с неподвижным газом поверхности покоящегося заряженного жидкого проводника в поле тяжести при учете поверхностного натяжения [13], [14].

Работа выполнена при поддержке Российского фонда фундаментальных исследований (проекты № 14-01-00056, 17-01-00037).

\section{Список литературы}

[1] Melcher J.R., Taylor G.I. // Annu. Rev. Fluid Mech. 1969. Vol. 1. P. 111-146.

[2] Saville D.A. // Annu. Rev. Fluid Mech. 1997. Vol. 29. P. 27 64.

[3] El-Sayed M.F. // Phys. Rev. E. 1999. Vol. 60. N 6. P. 7588 7591.

[4] Ширяева С.О. // ЖТФ. 2010. Т. 80. Вып. 2. С. 45-50.

[5] Tilley B.S., Petropoulos P.G., Papageorgiou D.T. // Phys. Fluid. 2001. Vol. 13. N 12. P. 3547-3563.

[6] Savettaseranee K., Papageorgiou D.T., Petropoulos P.G., Tilley B.S. // Phys. Fluid. 2003. Vol. 15. N 3. P. 641-652.

[7] Коровин В.М. // ЖТФ. 2011. Т. 81. Вып. 10. С. 12-19.

[8] Сивухин Д.В. Электричество. М.: Наука, 1983. 688 с.

[9] Ландау Л.Д., Лифшии, Е.М. Электродинамика сплошных сред. М.: Наука. 1992. 664 с. 
[10] Дразин Ф. Введение в теорию гидродинамической устойчивости. М.: Физматлит, 2005. 287 с.

[11] Ландау Л.Д., Либиии, Е.М. Гидродинамика. М.: Наука, 1988. $733 \mathrm{c}$

[12] Бреховских Л.М., Гончаров В.В. Введение в механику сплошных сред. М.: Наука, 1982. 335 с.

[13] Tonks L. // Phys. Rev. 1935. Vol. 48. P. 562-568.

[14] Френкель Я.И. // ЖЭТФ. 1936. Т. 6. Вып. 4. С. 347-350.

[15] Бэтчелор Джс. Введение в динамику жидкости. М.: Мир, 1973. $758 \mathrm{c}$.

[16] Таблищы физических величин. Под ред. Кикоина И.К. М.: Атомиздат, 1976. 1008 с. 